\title{
Observation of electric-quadrupole infrared transitions in water vapor
}

\author{
Alain Campargue $\odot,{ }^{1, *}$ Samir Kassi $\odot,{ }^{1}$ Andrey Yachmenev $\odot,{ }^{2,3}$ Aleksandra A. Kyuberis $\odot,{ }^{4}$ \\ Jochen Küpper, ${ }^{2,3,5}$ and Sergei N. Yurchenko ${ }^{6}$ \\ ${ }^{1}$ Université Grenoble Alpes, CNRS, LIPhy, 38000 Grenoble, France \\ ${ }^{2}$ Center for Free-Electron Laser Science, Deutsches Elektronen-Synchrotron DESY, Notkestraße 85, 22607 Hamburg, Germany \\ ${ }^{3}$ Center for Ultrafast Imaging, Universität Hamburg, Luruper Chaussee 149, 22761 Hamburg, Germany \\ ${ }^{4}$ Institute of Applied Physics, Russian Academy of Sciences, Ulyanov Street 46, Nizhny Novgorod 603950, Russia \\ ${ }^{5}$ Department of Physics, Universität Hamburg, Luruper Chaussee 149, 22761 Hamburg, Germany \\ ${ }^{6}$ Department of Physics and Astronomy, University College London, London WC1E 6BT, United Kingdom
}

(Received 8 January 2020; accepted 16 March 2020; published 28 April 2020)

\begin{abstract}
Molecular absorption of infrared radiation is generally due to rovibrational electric-dipole transitions. Electricquadrupole transitions may still occur, but they are typically a million times weaker than electric-dipole transitions, rendering their observation extremely challenging. In polyatomic or polar diatomic molecules, rovibrational quadrupole transitions have never been observed. Here, we report the direct detection of quadrupole transitions in water vapor. The detected quadrupole lines have intensity largely above the standard dipole intensity cutoff of spectroscopic databases and thus are important for accurate atmospheric and astronomical remote sensing.
\end{abstract}

DOI: 10.1103/PhysRevResearch.2.023091

Spectroscopic techniques in the IR domain have advanced significantly in recent years providing exciting opportunities for a generation of accurate and sensitive measurements. Quadrupole electronic and rovibrational transitions have been detected in a number of atoms $[1,2]$ and homonuclear diatomic molecules [3-5], respectively, for states that cannot undergo electric dipole transitions. Electric-quadrupole transitions are used for remote sensing of important diatomic molecules, such as hydrogen, oxygen, and nitrogen, in spectra of Earth's atmosphere and other environments [4,6,7]. When compared with dipole transitions, quadrupole transition rates are typically smaller by a factor ranging from $10^{8}$ in the IR to $10^{5}$ in the optical spectral domain. Such extremely long mean lifetimes against spontaneous emission of quadrupole excitations make them ideal candidates for next-generation atomic clocks [8], high-precision tests of molecular physics [9-11], and quantum information processing $[8,12]$.

In most heteronuclear diatomic and polyatomic molecules the rovibrational states can be excited both via dipole and quadrupole transitions. The dipole and quadrupole moment operators have distinct selection rules. The dipole moment connects states with different parity, while for the quadrupole transitions, the parity is conserved. Furthermore, states with rotational quantum numbers $J$ differing by \pm 2 can only be connected by a quadrupole transition. This difference in selection rules permits, in principle, the observation of

\footnotetext{
*Corresponding author: alain.campargue@univ-grenoble-alpes.fr
}

Published by the American Physical Society under the terms of the Creative Commons Attribution 4.0 International license. Further distribution of this work must maintain attribution to the author(s) and the published article's title, journal citation, and DOI. well-isolated quadrupole lines. Nevertheless, in practice the detection of quadrupole lines is made difficult by the fact that, even if transition frequencies differ, the quadrupole lines are drowned in the line profile of the much stronger dipole lines.

To the best of our knowledge, quadrupole transitions have never been detected in polyatomic molecules until now, although the quadrupole moment of some polyatomic molecules $\left(\mathrm{CO}_{2}, \mathrm{NO}_{2}\right.$, OCS $)$ have been measured using electric-field-gradient-induced birefringence method [13]. Currently, spectroscopic line lists of the major greenhouse gas absorbers such as water vapor and carbon dioxide, are limited to dipole transitions [14]. A highly accurate and complete characterization of rovibrational spectra of these molecules is needed for the modeling and understanding of Earth's atmosphere, climate, and many remote sensing experiments [15]. At present, adequate models of the water vapor absorption in the Earth's atmosphere incorporate many weak dipole lines including hot band transitions, minor water isotopologues contribution, and weak absorption continua [16]. Obviously, failure to include weak quadrupole transitions to the total opacities of atmospherically important species may contribute to systematic errors in remote sensing retrievals with a corresponding impact on atmospheric simulations.

Here, we report an observation of electric-quadrupole transitions in water vapor. This observation was made possible using a continuous wave diode laser cavity ring down spectroscopic (CRDS) technique to measure the water absorption spectrum near $1.3 \mu \mathrm{m}$. The detection and assignment of the measured spectral lines relied on high-accuracy predictions for both positions and intensities of the quadrupole $\mathrm{H}_{2}{ }^{16} \mathrm{O}$ transitions. It is important to stress that the detected quadrupole lines have intensity largely above the recommended dipole intensity cutoff of the standard spectroscopic databases and should thus be incorporated. 
CRDS is a high-sensitivity absorption technique that performs like a classical absorption spectroscopy, which would have a multikilometric absorption path length. The method consists of measuring the power decay rate (ring down) of a laser light trapped into a passive optical cavity, made of high-reflectivity semitransparent mirrors [17]. The presence of an absorbing gas in the cavity shortens the ring down time. From the variation of the decay rate with the laser frequency, one gets the absorption spectrum. Because of the extreme reflectivity of the mirrors (typically $\mathrm{R} \sim 99.997 \%$ ) sensitivities equivalent to several hundred kilometers in a classical absorption approach are routinely achieved and extremely weak absorptions are detected. A record absorption sensitivity, $\alpha_{\min } \approx 5 \times 10^{-13} \mathrm{~cm}^{-1}$, corresponding to a $2 \%$ light attenuation for the Earth-Moon distance, was achieved by CRDS [18], allowing for the detection of extremely weak quadrupole lines of $\mathrm{D}_{2}$ and $\mathrm{N}_{2}$ [19]. In the last two decades, the CRDS technique was extensively used to characterize weak dipole transitions of water vapor in the near-IR spectral domain [20]. Tens of thousands of new dipole transition lines were detected and assigned to various water isotopologues present in natural isotopic abundance. In the 1.6- and 1.25- $\mu \mathrm{m}$ transparency windows, lines of $\mathrm{HD}^{17} \mathrm{O}$ with a natural relative abundance of $1.158 \times 10^{-7}$ were detected [21] and dipole lines with intensities as weak as $1 \times 10^{-29} \mathrm{~cm} /$ molecule were measured [22].

At room temperature and typical sample cell pressures in the Torr range, most of quadrupole transitions of water molecule are masked by much stronger dipole transitions. In order to detect them in the CRDS spectrum, a highly accurate theoretical prediction of the quadrupole rovibrational spectrum is essential.

The calculations, detailed in Appendix A, employed a state-of-the-art variational approach based on high-level $a b$ initio electric dipole and quadrupole moment surfaces of $\mathrm{H}_{2} \mathrm{O}$. The robust variational approach TROVE (Theoretical RO-Vibrational Energies) [23,24] was used to solve the rovibrational Schrödinger equation for the motion of nuclei, based on exact kinetic energy operator [25,26] and recently reported accurate potential energy surface of $\mathrm{H}_{2} \mathrm{O}$ [25]. The electric-quadrupole moment surface was computed ab initio at the CCSD(T)/aug-cc-pVQZ level of theory in the frozen-core approximation using the CFOUR program package [26]. The quadrupole intensities and Einstein $A$ coefficients were calculated using the generalized approach RICHMOL for molecular dynamics in the presence of external electric fields [27]. The basis-set convergence of the rovibrational line positions was carefully examined. The remaining errors on the order of $0.01-0.6 \mathrm{~cm}^{-1}$ were still too significant to unambiguously identify quadrupole lines in the CRDS spectrum. To improve the accuracy, the computed rovibrational line positions were adjusted according to empirical values of the lower and upper state energy levels of $\mathrm{H}_{2}{ }^{16} \mathrm{O}$. These energies were carefully derived from the analysis of high-resolution spectroscopic data from more than a hundred experimental sources [28]. The average uncertainties on the order $10^{-3} \mathrm{~cm}^{-1}$ in the resulting line centers allowed to unambiguously identify quadrupole lines. Indeed, in the region of interest, with the sensitivity of the CRDS spectra under analysis, the spectral congestion is very high, due to dipole lines of $\mathrm{H}_{2}{ }^{16} \mathrm{O}$, water minor

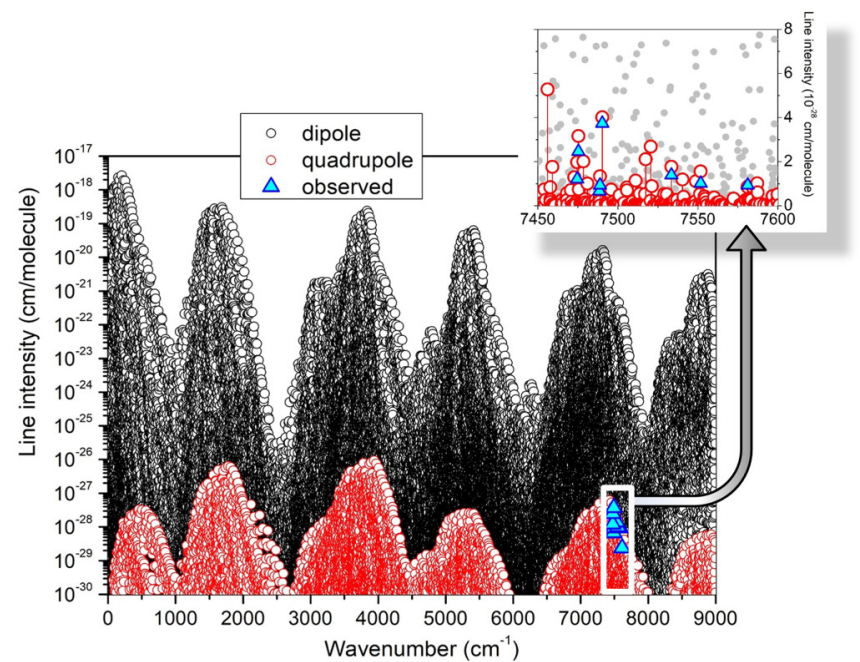

FIG. 1. Overview of the absorption line list of $\mathrm{H}_{2}{ }^{16} \mathrm{O}$ at $296 \mathrm{~K}$. The calculated electric-quadrupole spectrum [29] (red circles) is superimposed to the calculated electric-dipole spectrum (black circles). The CRDS measured quadrupole transitions are shown with blue triangles. The inset with intensities in linear scale shows a zoom of the $7450-7600 \mathrm{~cm}^{-1}$ region.

isotopologues, as well as impurities such as $\mathrm{CO}_{2}, \mathrm{NH}_{3}$ or $\mathrm{CH}_{4}$ present at ppm relative concentrations.

The overview of the calculated quadrupole line list of $\mathrm{H}_{2}{ }^{16} \mathrm{O}$ [29] is displayed in Fig. 1 superimposed to the dipole line list computed using an $a b$ initio dipole moment surface [30]. Both lists include all rovibrational transitions of $\mathrm{H}_{2}{ }^{16} \mathrm{O}$ with $J \leqslant 30$ and upper state energies below $10000 \mathrm{~cm}^{-1}$ with respect to the zero-point level. The general appearance of the quadrupole and dipole line lists is similar; the spectra consist of a succession of vibrational bands whose intensity decreases with the frequency. The maximum intensity values of quadrupole lines are typically seven orders of magnitude smaller than the dipole lines. Note that the strongest quadrupole lines near $4000 \mathrm{~cm}^{-1}$ have an intensity on the order of $10^{-26} \mathrm{~cm} /$ molecule, whereas the intensity cutoff the water dipole transitions included in the HITRAN database [14] is three orders of magnitude smaller $\left(10^{-29} \mathrm{~cm} / \mathrm{molecule}\right)$. Interestingly, the largest quadrupoleto-dipole intensity ratios of about $10^{-4}$ are predicted for the water transparency window at $2500 \mathrm{~cm}^{-1}$, dominated by transitions of the bending $v_{2}$ vibrational band. The complete quadrupole line list with transition frequencies up to $10000 \mathrm{~cm}^{-1}$ and $10^{-40} \mathrm{~cm} /$ molecule intensity cutoff is provided as Supplemental Material [29].

The CRDS recordings of natural water vapor were performed in the frequency range between 7408 and $7619 \mathrm{~cm}^{-1}$ at a pressure limited to 1.0 Torr and temperature maintained at 296 K. Details about the experimental setup and line parameters retrieval can be found in Appendix B. The noise equivalent absorption of the recordings, estimated as a root-meansquare deviation of the spectrum base line, is $\alpha_{\text {min }} \approx 5 \times$ $10^{-11} \mathrm{~cm}^{-1}$. In terms of line intensity, this converts to a detectivity threshold on the order of few $10^{-29} \mathrm{~cm} /$ molecule, well below the calculated intensities of the strongest quadrupole lines in the region (up to $6 \times 10^{-28} \mathrm{~cm} /$ molecule). 

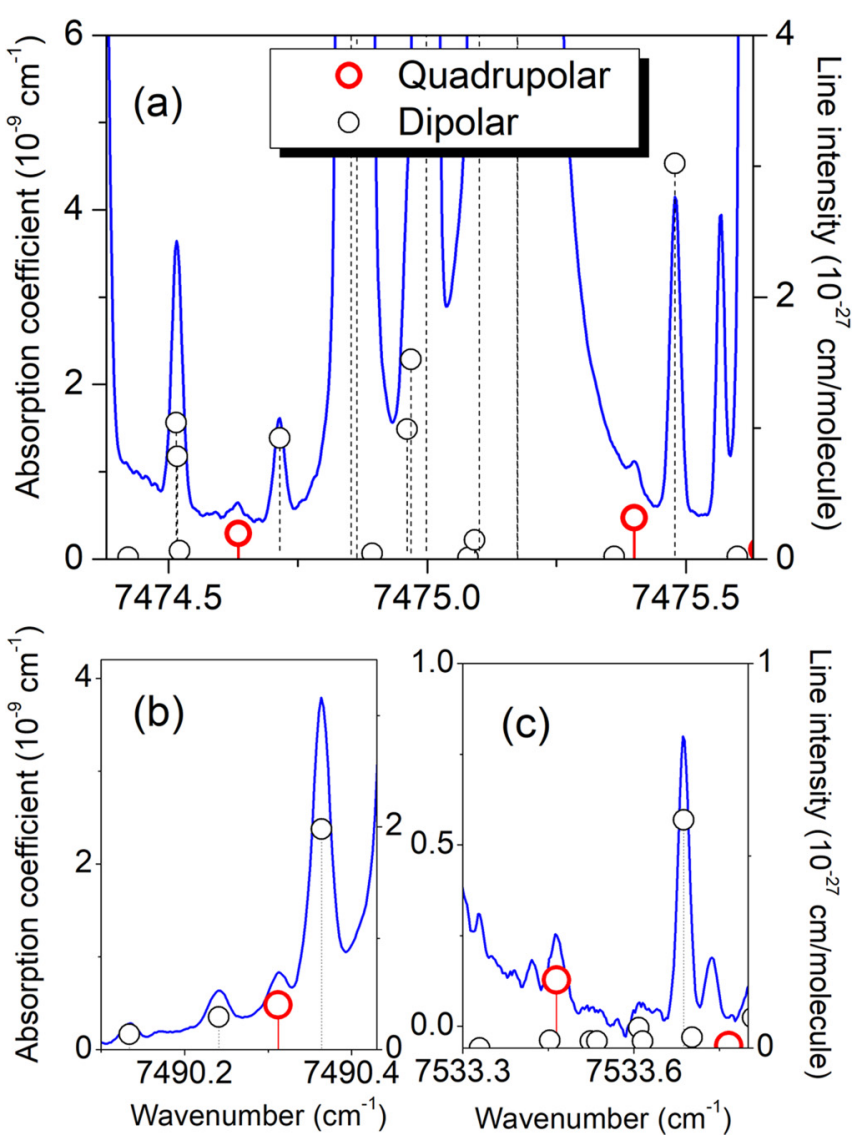

FIG. 2. Detection of electric-quadrupole lines of $\mathrm{H}_{2}{ }^{16} \mathrm{O}$ in the spectrum of water vapor recorded by CRDS at 1.0 Torr. On each panel, the calculated quadrupole stick spectrum of $\mathrm{H}_{2}{ }^{16} \mathrm{O}$ (red circles) and the dipole stick spectrum of water in natural isotopic abundance [14] (black circles) are superimposed to the CRDS absorption spectrum. Two quadrupole lines are detected near $7475 \mathrm{~cm}^{-1}$ [panel (a)], and one quadrupole line is detected near 7490.3 and $7533.5 \mathrm{~cm}^{-1}$ [panels (b) and (c), respectively]. The four detected quadrupole lines correspond to $\Delta J=2$ transitions of the $v_{1}+v_{3}$ band.

As illustrated in Fig. 2, the remarkable position and intensity coincidences of the calculated quadrupole lines to the recorded spectra leaves no doubt that $\mathrm{H}_{2}{ }^{16} \mathrm{O}$ quadrupole lines are detected. Among the 50 quadrupole lines predicted with sufficient intensity in the considered region, nine could be detected (blue triangles in Fig. 1), the others being hidden by stronger dipole lines. As a result, Table I lists the detected quadrupole lines with their measured positions and intensities obtained from the line profile fit described in Appendix B. The rovibrational assignments included in the table indicate that the detected lines belong to the $v_{1}+v_{3}$ band and correspond to $\Delta J=2$ transitions except one line corresponding to $\Delta J=1$. Experimental intensity values show a reasonable agreement with the calculated values (see inset in Fig. 1). Excluding the two weakest lines with large experimental uncertainties, the average measured/computed intensity ratio is $0.80(17)$.

This detection of quadrupole transitions in the water vapor spectrum opens perspectives for theoretical and experimental absorption spectroscopy of polyatomic molecules with expected impact in atmospheric and astronomical sciences. Despite being very weak, quadrupole absorption lines of water vapor are clearly above the background formed by the dipole spectrum and the water continuum and therefore must be included into modern atmospheric databases such as HITRAN [14] or ExoMol [31]. Water vapor, being one of the major interfering species for the trace gas analysis in air, missing quadrupole transitions may skew the optical measurement of the targeted species. In geosciences, a small, but measurable natural variation of abundance ratios of water minor isotopologues are used to trace various chemical and physical processes. In this context, absorption spectroscopy is an emerging method with specific advantages compared to conventional mass spectrometers [32-35]. Due to the small relative abundance of the minor isotopologues $\left(2 \times 10^{-3}\right.$ for $\mathrm{H}_{2}{ }^{18} \mathrm{O}$ and less than $4 \times 10^{-4}$ for HDO and $\mathrm{H}_{2}{ }^{17} \mathrm{O}$ ), their absorption lines are weak and any accidental coincidence between the target absorption line of the water minor isotopologue and an electric-quadrupole line of the main isotopologue $\left(\mathrm{H}_{2}{ }^{16} \mathrm{O}\right)$ will bias the retrieved abundances.

Similar biases may be encountered for metrological spectroscopic measurements of line intensities or line shapes, e.g., in the determination of the Boltzmann constant by Dopplerbroadening thermometry of a water line [36].

In the case of water vapor the envelopes of the strong quadrupole and dipole vibrational bands mostly coincide as

TABLE I. Assignment, transition frequencies, and intensities of the electric-quadrupole lines of the $v_{1}+v_{3}$ band of $\mathrm{H}_{2}{ }^{16} \mathrm{O}$ measured near $1.3 \mu \mathrm{m}$.

\begin{tabular}{|c|c|c|c|c|c|c|c|c|c|}
\hline \multicolumn{2}{|c|}{ Position $\left(\mathrm{cm}^{-1}\right)$} & \multicolumn{2}{|c|}{ Intensity (cm/molecule) } & \multicolumn{6}{|c|}{$J K_{a} K_{c}$} \\
\hline Meas. & Calc. & Meas. & Calc. & \multicolumn{3}{|c|}{ Upper level } & \multicolumn{3}{|c|}{ Lower level } \\
\hline 7474.6325 & 7474.6354 & $1.21 \times 10^{-28}$ & $1.98 \times 10^{-28}$ & 6 & 1 & 5 & 4 & 2 & 3 \\
\hline 7475.4020 & 7475.4005 & $2.46 \times 10^{-28}$ & $3.16 \times 10^{-28}$ & 5 & 2 & 4 & 3 & 1 & 2 \\
\hline 7488.5747 & 7488.5785 & $6.72 \times 10^{-29}$ & $3.23 \times 10^{-29}$ & 6 & 6 & 1 & 5 & 5 & 0 \\
\hline 7488.9183 & 7488.9215 & $9.34 \times 10^{-29}$ & $1.34 \times 10^{-28}$ & 7 & 0 & 7 & 5 & 1 & 5 \\
\hline 7490.3117 & 7490.3120 & $3.74 \times 10^{-28}$ & $4.02 \times 10^{-28}$ & 7 & 1 & 7 & 5 & 0 & 5 \\
\hline 7533.4649 & 7533.4642 & $1.39 \times 10^{-28}$ & $1.72 \times 10^{-28}$ & 7 & 2 & 6 & 5 & 1 & 4 \\
\hline 7551.7653 & 7551.7639 & $1.02 \times 10^{-28}$ & $1.56 \times 10^{-28}$ & 9 & 1 & 9 & 7 & 0 & 7 \\
\hline 7581.1247 & 7581.1172 & $9.32 \times 10^{-29}$ & $8.53 \times 10^{-29}$ & 10 & 0 & 10 & 8 & 1 & 8 \\
\hline 7613.8512 & 7613.8478 & $2.37 \times 10^{-29}$ & $1.10 \times 10^{-29}$ & 10 & 2 & 9 & 8 & 1 & 7 \\
\hline
\end{tabular}


a result of molecular symmetry and corresponding selection rules. This will not be the case in other important atmospheric species such as $\mathrm{CO}_{2}$ where, due to the linear structure of the molecule, different selection rules apply to quadrupole and dipole bands. Relatively strong quadrupole bands may be located in spectral regions where dipole absorption is weak, the so-called transparency windows. In these cases, the relative importance of quadrupole transitions will be more apparent.

This study paves the way for systematic computation of quadrupole spectra for all standard atmospheric molecules, e.g., $\mathrm{CO}_{2}, \mathrm{~N}_{2} \mathrm{O}, \mathrm{CH}_{4}, \mathrm{HCN}, \mathrm{CO}, \mathrm{HF}$. Owing to the weak character of the quadrupole spectra, systematic calculations across wide spectral ranges are thus required to evaluate the significance of quadrupole transitions for atmospheric, astrophysical, and industrial applications. After validation tests by high-sensitivity measurements, quadrupole transitions should be systematically incorporated into the most currently used spectroscopic databases.

\section{ACKNOWLEDGMENTS}

S.N.Y. acknowledges support from the UK Science and Technology Research Council (STFC) No. ST/R000476/1. A substantial part of the calculations was performed using high performance computing facilities provided by DiRAC for particle physics, astrophysics, and cosmology and supported by BIS National E-infrastructure capital Grant No. ST/K001590/1 and STFC Grants No. ST/H008861/1 and No. ST/H00887X/1. The work of A.A.K. was supported by the Foundation for the Advancement of Theoretical Physics and Mathematics BASIS. The work of A.Y. and J.K. has been supported by the Deutsche Forschungsgemeinschaft (DFG) through the clusters of excellence "Center for Ultrafast Imaging" (CUI, EXC 1074, ID 194651731) and "Advanced Imaging of Matter" (AIM, EXC 2056, ID 390715994). This research was supported in part through the Maxwell computational resources operated at Deutsches ElektronenSynchrotron (DESY), Hamburg, Germany.

\section{APPENDIX A: ELECTRONIC STRUCTURE CALCULATION}

For a water molecule in $C_{2 \mathrm{v}}(M)$ molecular symmetry group, the rank-2 quadrupole-moment tensor $Q$ transforms as $3 A_{1}+A_{2}+B_{1}+B_{2}$, where $A_{2}$ and $B_{1}$ components are zero and only two out of three $A_{1}$-symmetry components are necessary, because the quadrupole tensor is traceless, i.e., $Q_{A_{1}}^{(1)}+Q_{A_{1}}^{(2)}+Q_{A_{1}}^{(3)}=0$. In total, three symmetry-adapted components are sufficient to describe the traceless quadrupole tensor of water, these can be constructed as

$$
\begin{aligned}
Q_{A_{1}}^{(i)} & =\sum_{\alpha, \beta=x, y, z} S_{i, \alpha} S_{i, \beta} Q_{\alpha, \beta} \quad \text { for } i=1,2, \\
Q_{B_{2}} & =\sum_{\alpha, \beta=x, y, z} S_{1, \alpha} S_{2, \beta} Q_{\alpha, \beta},
\end{aligned}
$$

where

$$
\mathbf{S}_{i}=\frac{\mathbf{r}_{\mathrm{H}_{i}}-\mathbf{r}_{\mathrm{O}}}{r_{\mathrm{OH}_{i}}},
$$

$Q_{\alpha, \beta}$ denotes Cartesian components of the quadrupole moment tensor, and $r_{\mathrm{OH}_{i}}$ is the $\mathrm{O}-\mathrm{H}_{i}$ bond distance. The quadrupole moment tensor for water was computed $a b$ initio at the CCSD(T)/aug-cc-pVQZ [37,38] level of theory in the frozen-core approximation. Calculations were performed on a grid of about 2000 different molecular geometries covered the wave-number energy range up to $30000 \mathrm{~cm}^{-1}$ above the equilibrium energy. Analytic CCSD(T) gradient method was used [39], as implemented in the CFOUR program package [26]. The symmetrized components in Eq. (A1) were parametrized using fourth order symmetry-adapted power series expansions through least-squares fittings.

\section{Rovibrational energies and wave functions}

The variational program TROVE [23] has been used to solve the rovibrational Schrödinger equation in the ground electronic state. A new implementation of the exact kinetic energy (EKE) operator developed for this study was used. The EKE is based on the bisector embedding for triatomic molecules [40,41]. Accurate potential energy surface [25], electric dipole moment surface [30] and electric-quadrupole moment surface (this work) were employed.

TROVE uses a multilayer contraction scheme (see, for example, [42]). At step 1, stretching and bending primitive basis functions are constructed numerically by solving the corresponding one-dimensional (1D) Schrödinger equations. The two equivalent stretching equations are solved on a grid of 2000 points using the Numerov-Cooley approach [43,44]. For the bending solution, the associated Laguerre polynomials are used, which are optimized for the corresponding 1D Schrödinger equation on a grid of 3000 points. The bending 1D Hamiltonian includes the $k^{2}$-dependent centrifugal distortion term. The details of the model will be published elsewhere [45]. The model 1D Hamiltonian for a given mode (stretch or bend) is constructed by setting the other mode to its equilibrium value. At step 2 the $1 \mathrm{D}$ basis functions are then used to solve two reduced problems for the $2 \mathrm{D}$ stretching and 1D bending reduced Hamiltonians variationally constructed by averaging the $3 \mathrm{D}$ vibrational $(J=0)$ Hamiltonian over the ground state basis functions as follows:

$$
\begin{gathered}
\hat{H}^{(2 \mathrm{D})}=\left\langle 0_{3}|\hat{H}| 0_{3}\right\rangle, \\
\hat{H}^{(1 \mathrm{D})}=\left\langle 0_{1} 0_{2}|\hat{H}| 0_{1} 0_{2}\right\rangle,
\end{gathered}
$$

where the $\left|v_{i}\right\rangle$ band are the stretching $(i=1,2)$ and bending $(i=3)$ vibrational basis functions with $v=0$.

The eigenfunctions of these problems are symmetrized using an automatic symmetry adaptation technique [24] and form a $3 \mathrm{D}$ vibrational basis set for the $J=0$ Hamiltonian for step 3 . The $C_{2 \mathrm{v}}(M)$ molecular group symmetry is used to classify the irreducible representations. The eigenfunctions of the $(J=0)$ 3D Hamiltonian are then used to form a rovibrational basis set for all $J>0$ calculations, where the rotational part is constructed from symmetrized rigid rotor functions [24]. Our primitive basis set comprised 18 and 48 functions for the stretching and bending modes, respectively. The atomic masses were used. An $E / h c=40000 \mathrm{~cm}^{-1}$ energy cutoff was used to contract the $J=0$ eigenfunctions. All energies and eigenfunctions up to $J \leqslant 30$ were generated and used to 
TABLE II. Comparison of empirical (Obs.) [28] and calculated (Calc.) energy term values of $\mathrm{H}_{2}{ }^{16} \mathrm{O}$ up to $9000 \mathrm{~cm}^{-1}$.

\begin{tabular}{|c|c|c|c|c|c|c|c|c|c|c|c|}
\hline$v_{1}$ & $v_{2}$ & $v_{3}$ & $\begin{array}{c}\text { Obs. } \\
\left(\mathrm{cm}^{-1}\right)\end{array}$ & $\begin{array}{l}\text { Calc. } \\
\left(\mathrm{cm}^{-1}\right)\end{array}$ & $\begin{array}{l}\text { Obs.-Calc. } \\
\quad\left(\mathrm{cm}^{-1}\right)\end{array}$ & $v_{1}$ & $v_{2}$ & $v_{3}$ & $\begin{array}{l}\text { Obs. } \\
\left(\mathrm{cm}^{-1}\right)\end{array}$ & $\begin{array}{l}\text { Calc. } \\
\left(\mathrm{cm}^{-1}\right)\end{array}$ & $\begin{array}{c}\text { Obs.-Calc. } \\
\left(\mathrm{cm}^{-1}\right)\end{array}$ \\
\hline 0 & 0 & 0 & 0.000 & 0.000 & 0.000 & 2 & 0 & 0 & 7249.817 & 7250.459 & -0.642 \\
\hline 0 & 0 & 1 & 1594.746 & 1594.748 & -0.002 & 1 & 1 & 0 & 7445.366 & 7445.366 & 0.000 \\
\hline 0 & 0 & 2 & 3151.630 & 3151.640 & -0.010 & 0 & 0 & 5 & 7542.372 & 7542.423 & -0.050 \\
\hline 1 & 0 & 0 & 3657.053 & 3657.159 & -0.106 & 1 & 0 & 3 & 8273.976 & 8274.070 & -0.094 \\
\hline 0 & 1 & 0 & 3755.929 & 3756.058 & -0.129 & 0 & 1 & 3 & 8373.851 & 8373.952 & -0.101 \\
\hline 0 & 0 & 3 & 4666.790 & 4666.789 & 0.001 & 0 & 2 & 1 & 8761.582 & 8762.077 & -0.495 \\
\hline 1 & 0 & 1 & 5234.976 & 5235.077 & -0.101 & 2 & 0 & 1 & 8806.999 & 8807.549 & -0.550 \\
\hline 0 & 1 & 1 & 5331.267 & 5331.374 & -0.107 & 0 & 0 & 6 & 8869.950 & 8870.160 & -0.210 \\
\hline 0 & 0 & 4 & 6134.015 & 6134.017 & -0.002 & 1 & 1 & 1 & 9000.410 & 9000.410 & 0.000 \\
\hline 1 & 0 & 2 & 6775.094 & 6775.186 & -0.093 & 1 & 0 & 4 & 9724.313 & 9724.313 & 0.000 \\
\hline 0 & 1 & 2 & 6871.520 & 6871.622 & -0.102 & 0 & 1 & 4 & 9833.583 & 9833.686 & -0.103 \\
\hline 0 & 2 & 0 & 7201.540 & 7202.099 & -0.559 & & & & & & \\
\hline
\end{tabular}

produce dipole and quadrupole line lists for water. The final TROVE eigenfunctions are given in a sum-of-product form:

$$
\left|J, m_{J}, l\right\rangle=\sum_{v, k} c_{v, k}^{(J, l)}|v\rangle\left|J, m_{J}, k\right\rangle
$$

where $m_{J}$ is a projection of the angular momentum on the laboratory $Z$ axis, $k$ is a projection on the molecular $z$ axis, $l$ is a counting state number, $v$ is a generic vibrational $(J=0)$ quantum number, and $\mathrm{c}_{v, k}^{(J, l)}$ is an expansion eigencoefficient. In order to improve the calculated line positions, empirical energies of $\mathrm{H}_{2}{ }^{16} \mathrm{O}$ [28] were used to replace the TROVE energies where available. In this replacement we took advantage of the two-file structure of the ExoMol line list consisting of a states file and a transition file [31,46].

As an illustration of the quality of the present calculations, Table II shows a comparison of the calculated energy term values (before substitution) with the experimental IUPAC values [28]. The differences can be attributed to the artefacts of the conversion of the empirical photoemission spectroscopy of $\mathrm{H}_{2} \mathrm{O}$ [25] to the analytical representation used in TROVE.

\section{Quadrupole spectrum simulations}

The quadrupole spectrum has been simulated using the variational approach RichMol [27,47], designed for calculations of molecular rovibrational dynamics in the presence of external electromagnetic fields. The transition probability due to the electric-quadrupole interaction from a set of $(2 J+1)$ degenerate initial rovibrational states $|J, m, l\rangle$ into a set of $\left(2 J^{\prime}+1\right)$-degenerate final rovibrational states $\left|J^{\prime}, m^{\prime}, l^{\prime}\right\rangle$ is given by

$$
\begin{aligned}
& P_{Q}\left(J^{\prime}, l^{\prime} \leftarrow J, l\right) \\
& \quad=\sum_{m^{\prime}=-J^{\prime}}^{J^{\prime}} \sum_{m=-J}^{J} \sum_{A, B=X, Y, Z}\left|\left\langle J^{\prime}, m^{\prime}, l^{\prime}\left|Q_{A, B}\right| J, m, l\right\rangle\right|^{2} .
\end{aligned}
$$

The rovibrational matrix elements of a traceless quadrupole tensor operator $Q_{A, B}$ in the laboratory frame $(A, B=X, Y, Z)$ can generally be represented in a contracted tensor form

$$
\left\langle J^{\prime}, m^{\prime}, l^{\prime}\left|Q_{A, B}\right| J, m, l\right\rangle=M_{A, B}^{\left(J^{\prime}, m^{\prime}, J, m\right)} K^{\left(J^{\prime}, l^{\prime}, J, l\right)},
$$

with

$$
\begin{aligned}
M_{A, B}^{\left(J^{\prime}, m^{\prime}, J, m\right)}= & (-1)^{m^{\prime}} \sqrt{(2 J+1)\left(2 J^{\prime}+1\right)} \\
& \times \sum_{\sigma=-2}^{2}[U]_{A, B, \sigma}^{-1}\left(\begin{array}{ccc}
J & 2 & J^{\prime} \\
m & \sigma & -m^{\prime}
\end{array}\right)
\end{aligned}
$$

and

$$
\begin{aligned}
K^{\left(J^{\prime}, l^{\prime}, J, l\right)}= & \sum_{k^{\prime}, v^{\prime}} \sum_{k, v}\left[c_{v^{\prime}, k^{\prime}}^{\left(J^{\prime}, l^{\prime}\right)}\right]^{*}\left[c_{v, k}^{(J, l)}\right](-1)^{k^{\prime}} \sum_{\sigma=-2}^{2} \sum_{\alpha, \beta=x, y, z} \\
& \times\left(\begin{array}{ccc}
J & 2 & J^{\prime} \\
k & \sigma & -k^{\prime}
\end{array}\right) U_{\sigma, \alpha \beta}\left\langle v^{\prime}\left|Q_{\alpha, \beta}\right| v\right\rangle .
\end{aligned}
$$

Here, matrix $U$ defines the transformation of the traceless symmetric rank-2 tensor from Cartesian to spherical tensor form (see, e.g., Table I in Ref. [27] for explicit expression). The rovibrational wave-function coefficients $\mathrm{c}_{v, k}^{(J, l)}$ and vibrational matrix elements of quadrupole tensor operator $\left\langle v^{\prime}\left|\mathrm{Q}_{\alpha, \beta}\right| v\right\rangle$ in the molecular frame were calculated using the TROVE approach, as described in the previous section. Using Eqs. (A6)-(A8), the expression for the rovibrational transition probability [in Eq. (A6)] can be derived as

$$
\begin{aligned}
& P_{Q}\left(J^{\prime}, l^{\prime} \leftarrow J, l\right) \\
& \quad=\left[K^{\left(J^{\prime}, l^{\prime}, J, l\right)}\right]^{2} \sum_{m^{\prime}=-J^{\prime}}^{J^{\prime}} \sum_{m=-J}^{J} \sum_{A, B=X, Y, Z}\left|M_{A, B}^{\left(J^{\prime}, m^{\prime}, J, m\right)}\right|^{2} \\
& \quad=3.8(2 J+1)\left(2 J^{\prime}+1\right)\left[K^{\left(J^{\prime}, l^{\prime}, J, l\right)}\right]^{2} .
\end{aligned}
$$

The expression for the integrated absorption coefficient of the quadrupole transition in units $\mathrm{cm} /$ molecule reads as

$I_{Q}\left(J^{\prime}, l^{\prime} \leftarrow J, l\right)=\frac{4 \pi^{5} v^{3} e^{-\frac{E_{J, l}}{k T}}\left(1-e^{-\frac{h c v}{k T}}\right)}{\left(4 \pi \epsilon_{0}\right) 5 h c Z(T)} P_{Q}\left(J^{\prime}, l^{\prime} \leftarrow J, l\right)$,

where $v=\left(E_{J^{\prime}, l^{\prime}}-E_{J, l}\right) / h c$ is the transition frequency with $E_{J^{\prime}, l^{\prime}}$ and $E_{J, l}$ being upper and lower state energies, respectively, and $Z(T)$ is the partition function. Here we used the value of $Z(T=296 \mathrm{~K})=174.5813$. 


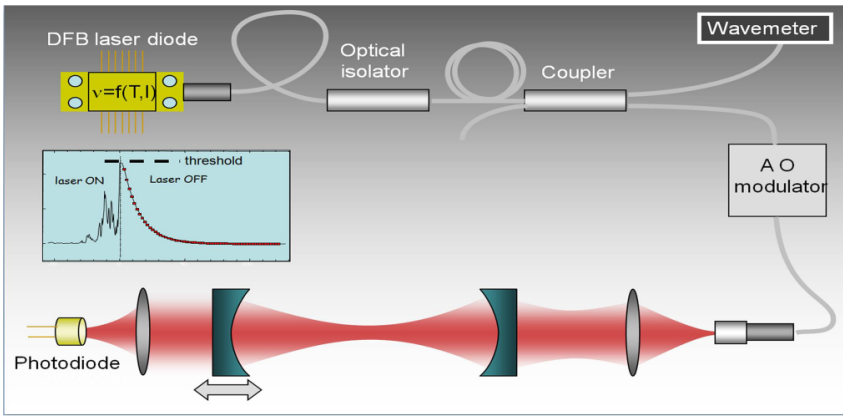

FIG. 3. Scheme of the CRD spectrometer. The different components include a distributed feedback diode laser (DFB), an optical isolator, a fiber coupler, an acousto-optic (AO) modulator and a photodiode. The inset shows the time dependence of the light intensity measured by the photodiode: after a build-up period, the light intensity reaches a threshold value which triggers the switch off of the AO modulator. The ring down time is then measured from the exponential decay of the light intensity.

\section{APPENDIX B: CRDS SETUP AND LNE PARAMTER RETRIVIAL}

The CRDS spectrum of water at room temperature was recorded from 7408 to $7619 \mathrm{~cm}^{-1}$ with a fibred CRDS spectrometer using distributed feed-back (DFB) diode lasers as light sources. A sketch of the experimental arrangement is presented in Fig. 3. The stainless steel high finesse cell (HFC) $\left(l=1.42 \mathrm{~m}, \Phi_{\text {in }}=11.7 \mathrm{~mm}\right)$ is fitted by a pair of supermirrors ( $\sim 99.998 \%$ reflectivity) giving rise to ring down times on the order of $200 \mu$ s. The whole spectral region was continuously covered by means of six fibered DFB lasers. The latter were supplied with a constant current of $140 \mathrm{~mA}$, tuned over about $35 \mathrm{~cm}^{-1}$ by a temperature sweep of -10 to $60{ }^{\circ} \mathrm{C}$. About 40 ring down events were averaged for each spectral data point, distant by approximately $2 \times 10^{-3} \mathrm{~cm}^{-1}$. A complete DFB temperature scan was achieved within 65 min. Each time a longitudinal mode of the HFC was excited by the laser frequency, an exponential ring down (RD) event was induced by switching off the laser beam using an acoustooptic modulator. In order to excite the HFC mode whatever the laser frequency, the HFC length was modulated over one free spectral range by a piezotube supporting the output mirror [17]. RD times, $\tau$, were measured by fitting with a purely decreasing exponential function the transmission signal measured with an InGaAs photodiode. The obtained loss rate, $1 / c \tau$, is the sum of the loss rate of the evacuated cell, $1 / c \tau_{0}$, and of the absorption coefficient, $\alpha(v)$, of the gas:

$$
\frac{1}{c \tau(v)}=\frac{1}{c \tau_{0}(v)}+\alpha(v),
$$

where $c$ is the speed of light.

The spectra were recorded at 1.0 Torr. The pressure was continuously monitored by a capacitance gauge (Baratron) and the temperature was stabilized at about 296 K. Each $35-\mathrm{cm}^{-1}$ wide spectrum, corresponding to one DFB recording, was calibrated independently on the basis of the wavenumber values provided by a Fizeau type wave meter (WSU30 Highfinesse, 5-MHz resolution and 20-MHz accuracy).
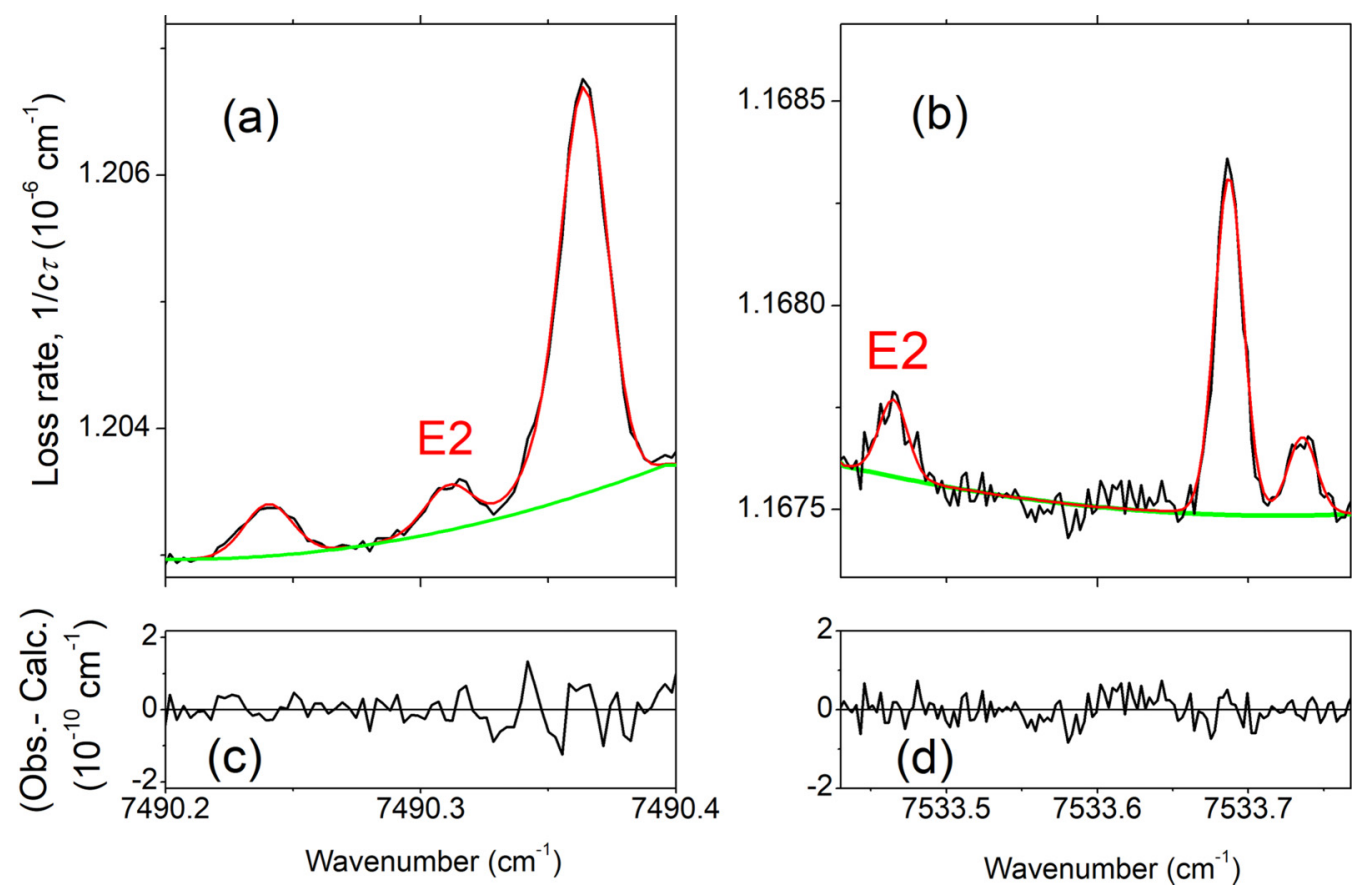

FIG. 4. Examples of line profile fitting of electric-quadrupole lines of $\mathrm{H}_{2}{ }^{16} \mathrm{O}$. On the upper panels (a) and (b), a spectrum simulation (red line) is superimposed to the CRDS spectrum (black line). The simulated spectrum was obtained as a sum of fitted Voigt profiles and a fitted baseline assumed to be a quadratic polynomial of the wave number (green line). The electric-quadrupole lines at 7490.311 and $7533.465 \mathrm{~cm}^{-1}$ [marked "E2" on panels (a) and (b), respectively] are detected near weak electric-dipole lines. The lower panels (c) and (d) show the corresponding differences between the CRDS and fitted spectra in $10^{-10} \mathrm{~cm}^{-1}$ units. 
The calibration was checked and refined using the reference line positions of $\mathrm{H}_{2} \mathrm{O}$ from the HITRAN database [14].

The line centers and line intensities were obtained by using a homemade interactive least-squares multilines fitting program written in LabVIEW. As the DFB line width (due to the frequency jitter) is much smaller than the Doppler broadening (1-5 MHz compared to $1 \mathrm{GHz}$ ), the contribution of the apparatus function to the observed profiles was neglected. The line parameter derivation consisted of choosing narrow spectral intervals with (overlapping) lines that could be fitted independently. For each interval, the centers, integrated absorption coefficients, and pressure broadenings of the lines, and a baseline (assumed to be a quadratic function of the wave-number) were provided by the fitting procedure. The Doppler broadening was fixed according to the mass and temperature. Figure 4 illustrates the achieved spectrum reproduction close to the noise level for two electric-quadrupole lines.
The line intensity, $S_{\nu_{0}}$ of a rovibrational transition centered at $v_{0}$, was obtained from the integrated absorption coefficient, $A_{v_{0}}\left(\mathrm{~cm}^{-2} /\right.$ molecule $)$ :

$$
A_{\nu_{0}}=\int_{\text {line }} \alpha_{\nu} d v=S_{\nu_{0}}(T) N,
$$

where $v$ is the wave number in $\mathrm{cm}^{-1}, \alpha_{v}$ is the absorption coefficient in $\mathrm{cm}^{-1}$ obtained from the cavity ring down time [Eq. (B1)], and $N$ is the molecular concentration in molecule $/ \mathrm{cm}^{3}$ obtained from the measured pressure and temperature values: $P=N k T$.

The retrieved centers and intensities of the nine quadrupolar lines are given in Table I. As a result of the weakness of the considered lines and strong line overlapping, the error bars on the fitted line centers are estimated to be $2-3 \times 10^{-3} \mathrm{~cm}^{-1}$, while the uncertainty on the experimental intensities ranges between 20 and $60 \%$.
[1] C. F. Roos, M. Chwalla, K. Kim, M. Riebe, and R. Blatt, 'Designer atoms' for quantum metrology, Nature (London) $\mathbf{4 4 3}$, 316 (2006).

[2] S. S. Kondov, C.-H. Lee, K. H. Leung, C. Liedl, I. Majewska, R. Moszynski, and T. Zelevinsky, Molecular lattice clock with long vibrational coherence, Nat. Phys. 15, 1118 (2019).

[3] G. Herzberg, Quadrupole rotation-vibration spectrum of the hydrogen molecule, Nature (London), 163, 170 (1949).

[4] L. S. Rothman and A. Goldman, Infrared electric quadrupole transitions of atmospheric oxygen, Appl. Opt. 20, 2182 (1981).

[5] A. Campargue, S. Kassi, K Pachucki, and J. Komasa, The absorption spectrum of $\mathrm{H}_{2}$ : CRDS measurements of the (2-0) band, review of the literature data and accurate $a b$ initio line list up to $35000 \mathrm{~cm}^{-1}$, Phys. Chem. Chem. Phys. 14, 802 (2012).

[6] A. Goldman, J. Reid, and L. S. Rothman, Identification of electrical quadrupole $\mathrm{O}_{2}$ and $\mathrm{N}_{2}$ lines in the infrared atmospheric absorption spectrum due to the vibration-rotation fundamentals, Geophys. Res. Lett. 8, 77 (1981).

[7] K. H. Baines, M. E. Mickelson, L. E. Larson, and D. W. Ferguson, The abundances of methane and ortho/para hydrogen on Uranus and Neptune: Implications of New Laboratory 4-0 $\mathrm{H}_{2}$ quadrupole line parameters, Icarus 114, 328 (1995).

[8] A. D. Ludlow, M. M. Boyd, Jun Ye, E. Peik, and P. O. Schmidt, Optical atomic clocks, Rev. Mod. Phys. 87, 637 (2015).

[9] J. C. Berengut, V. A. Dzuba, and V. V. Flambaum, Enhanced Laboratory Sensitivity to Variation of the Fine-Structure Constant Using Highly Charged Ions, Phys. Rev. Lett. 105, 120801 (2010).

[10] T. Pruttivarasin, M. Ramm, S. G. Porsev, I. I. Tupitsyn, M. S. Safronova, M. A. Hohensee, and H. Häffner, MichelsonMorley analogue for electrons using trapped ions to test Lorentz symmetry, Nature (London) 517, 592 (2015).

[11] M. Quack, J. Stohner, and M. Willeke, High-resolution spectroscopic studies and theory of parity violation in chiral molecules, Annu. Rev. Phys. Chem. 59, 741 (2008).

[12] H. Haeffner, C. F. Roos, and R. Blatt, Quantum computing with trapped ions, Phys. Rep. 469, 155 (2008).
[13] A. D. Buckingham, R. L. Disch, and D. A. Dunmur, The quadrupole moments of some simple molecules, J. Am. Chem. Soc. 90, 3104 (1968).

[14] I. E. Gordon et al., The HITRAN2016 molecular spectroscopic database, J. Quant. Spectrosc. Radiat. Transfer. 203, 3 (2017).

[15] O. L. Polyansky, N. F. Zobov, I. I. Mizus, L. Lodi, S. N. Yurchenko, J. Tennyson, A. G. Császár, and O. V. Boyarkin, Global spectroscopy of the water monomer, Philos. Trans. Royal Soc. London A 370, 2728 (2012).

[16] B. A. Fomin and V. A. Falaleeva, Recent progress in spectroscopy and its effect on line-by-line calculations for the validation of radiation codes for climate models, Atmos. Ocean. Opt. 22, 626 (2009).

[17] D. Romanini, A. A. Kachanov, N. Sadeghi, and F. Stoeckel, CW cavity ring down spectroscopy, Chem. Phys. Lett. 264, 316 (1997).

[18] S. Kassi and A. Campargue, Cavity ring down spectroscopy with $5 \times 10^{-13} \mathrm{~cm}^{-1}$ sensitivity, J. Chem. Phys. 137, 234201 (2012).

[19] S. Kassi, I. E. Gordon, and A. Campargue, First detection of transitions in the second quadrupole overtone band of nitrogen near $1.44 \mu \mathrm{m}$ by CW-CRDS with $6 \times 10^{-13} \mathrm{~cm}^{-1}$ sensitivity, Chem. Phys. Lett. 582, 6 (2013).

[20] S. N. Mikhailenko, S. Kassi, D. Mondelain, R. R. Gamache, and A. Campargue, A spectroscopic database for water vapor between 5850 and $8340 \mathrm{~cm}^{-1}$, J. Quant. Spectrosc. Radiat. Transfer. 179, 198 (2016).

[21] O. Leshchishina, S. Mikhailenko, D. Mondelain, S. Kassi, and A. Campargue, An improved line list for water vapor in the $1.5 \mu \mathrm{m}$ transparency window by highly sensitive CRDS between 5852 and $6607 \mathrm{~cm}^{-1}$, J. Quant. Spectrosc. Radiat. Transfer. 130, 69 (2013).

[22] A. Campargue, S. N. Mikhailenko, B. Guillo Lohan, E. V. Karlovets, D. Mondelain, and S. Kassi, The absorption spectrum of water vapor in the $1.25 \mu \mathrm{m}$ atmospheric window $\left(7911-8337 \mathrm{~cm}^{-1}\right)$, J. Quant. Spectrosc. Radiat. Transfer. 157, 135 (2015). 
[23] S. N. Yurchenko, W. Thiel, and P. Jensen, Theoretical ROVibrational energies (TROVE): A robust numerical approach to the calculation of rovibrational energies for polyatomic molecules, J. Mol. Spectrosc. 245, 126 (2007).

[24] S. N. Yurchenko, A. Yachmenev, and R. I. Ovsyannikov, Symmetry adapted ro-vibrational basis functions for variational nuclear motion: TROVE approach, J. Chem. Theory Comput. 13, 4368 (2017).

[25] I. I. Mizus, A A. Kyuberis, N. F. Zobov, V. Yu. Makhnev, O. L. Polyansky, and J. Tennyson, High accuracy water potential energy surface for the calculation of infrared spectra, Philos. Trans. Royal Soc. London A 376, 20170149 (2018).

[26] CFOUR, Coupled-Cluster techniques for Computational Chemistry, a quantum chemical program package written by J. F. Stanton et al. and the integral packages MOLECULE (J. Almlöf and P. R. Taylor), PROPS (P. R. Taylor), ABACUS (T. Helgaker et al.), and ECP routines by A.V. Mitin and C. van Wüllen, for the current version, see http://www.cfour.de (2018).

[27] A. Owens and A. Yachmenev, RichMol: A general variational approach for rovibrational molecular dynamics in external electric fields, J. Chem. Phys. 148, 124102 (2018).

[28] J. Tennyson et al., IUPAC critical evaluation of the rotationalvibrational spectra of water vapor. Part III: Energy levels and transition wavenumbers for $\mathrm{H}_{2}{ }^{16} \mathrm{O}$, J. Quant. Spectrosc. Radiat. Transfer. 117, 29 (2013).

[29] See Supplemental Material at http://link.aps.org/supplemental/ 10.1103/PhysRevResearch.2.023091 for the calculated electricquadrupole line list of $\mathrm{H}_{2}{ }^{16} \mathrm{O}$.

[30] E. K. Conway, A. A. Kyuberis, O. L. Polyansky, J. Tennyson, and N. F. Zobov, A highly accurate $a b$ initio dipole moment surface for the ground electronic state of water vapor for spectra extending into the ultraviolet, J. Chem. Phys. 149, 084307 (2018).

[31] J. Tennyson and S. N. Yurchenko, ExoMol: Molecular line lists for exoplanet and other atmospheres, Mon. Not. R. Astron. Soc. 425, 21 (2012).

[32] E. S. F. Berman, N. E. Levin, A. Landais, S. Li, and T. Owano, Measurement of $\delta^{18} \mathrm{O}, \delta^{17} \mathrm{O}$, and ${ }^{17} \mathrm{O}$-excess in water by offaxis integrated cavity output spectroscopy and isotope ratio mass spectrometry, Anal. Chem. 85, 10392 (2013).

[33] E. R. T. Kerstel and H. A. J. Meijer, Optical Isotope Ratio Measurements in Hydrology (Chapter 9), in Isotopes in the Water Cycle: Past, Present and Future of A Developing Science, edited by P. K. Aggarwal, J. Gat, and K. Froehlich (IAEA Hydrology Section, Kluwer, Dordrecht, 2005), pp. 109-124.
[34] J. B. McManus, D. D. Nelson, and M. S. Zahniser, Design and performance of a dual-laser instrument for multiple isotopologues of carbon dioxide and water, Opt. Express 23, 6569 (2015).

[35] E. J. Steig, V. Gkinis, A. J. Schauer, S. W. Schoenemann, K. Samek, J. Hoffnagle, K. J. Dennis, and S. M. Tan, Calibrated high-precision o measurements using laser-current tuned cavity ring-down spectroscopy, Atmos. Meas. Tech. 6, 10191 (2013).

[36] L. Moretti, A. Castrillo, E. Fasci, M. D. De Vizia, G. Casa, G. Galzerano, A. Merlone, P. Laporta, and L. Gianfrani, Determination of the Boltzmann Constant by Means of Precision Measurements of Line Shapes at $1.39 \mu \mathrm{m}$, Phys. Rev. Lett. 111, 060803 (2013).

[37] T. H. Dunning, Gaussian basis sets for use in correlated molecular calculations. I. The atoms boron through neon and hydrogen, J. Chem. Phys. 90, 1007 (1989).

[38] R. A. Kendall, T. H. Dunning Jr., and R. J. Harrison, Electron affinities of the first-row atoms revisited. Systematic basis sets and wave functions, J. Chem. Phys. 96, 6796 (1992).

[39] G. E. Scuseria and E, Analytic evaluation of energy gradients for the singles and doubles coupled cluster method including perturbative triple excitations: Theory and applications to FOOF and $\mathrm{Cr}_{2}$, J. Chem. Phys. 94, 442 (1991).

[40] S. Carter, N. C. Handy, and B. T. Sutcliffe, A variational method for the calculation of rovibrational levels of any triatomic molecule, Mol. Phys. 49, 745 (1983).

[41] B. T. Sutcliffe and J. Tennyson, A general treatment of vibration-rotation coordinates for triatomic molecules, Intern. J. Quantum. Chem. 39, 183 (1991).

[42] J. Tennyson and S. N. Yurchenko, The ExoMol project: Software for computing large molecular line lists, Intern. J. Quantum Chem. 117, 92 (2017).

[43] J. W. Cooley, An improved eigenvalue corrector formula for solving the Schrödinger equation for central fields, Math. Comp. 15, 363 (1961).

[44] B. V. Noumerov, A method of extrapolation of perturbations, Mon. Not. R. Astron. Soc. 84, 592 (1924).

[45] S. N. Yurchenko and T. Mellor (unpublished).

[46] J. Tennyson et al., The ExoMol database: Molecular line lists for exoplanet and other hot atmospheres, J. Mol. Spectrosc. 327, 73 (2016).

[47] A. Yachmenev, L. V. Thesing, and J. Küpper, Laser-induced dynamics of molecules with strong nuclear quadrupole coupling, J. Chem. Phys. 151, 244118 (2019). 